\title{
Malformación adenomatoidea quística congénita
}

\author{
PILAR ANTÓN-MARTÍN¹, M. TERESA CUESTA-RUBIO¹, M. FERNANDA LÓPEZ-GONZÁLEZ, \\ ROBERTO ORTIZ-MOVILLA ${ }^{1}$, M. LUISA LORENTE-JAREÑO², \\ ESTHER LÓPEZ-RODRÍGUEZ², LUCÍA CABANILLAS-VILAPLANA ${ }^{1}$ \\ 1. Servicio de Pediatría. Unidad de Neonatología. Hospital Universitario de Getafe. Madrid. España. \\ 2. Servicio de Radiodiagnóstico. Unidad de Radiología Pediátrica. Hospital Universitario de Getafe. Madrid. España.
}

\begin{abstract}
Congenital cystic adenomatous malformation

Introduction: Congenital Cystic Adenomatous Malformation (CCAM) is an infrequent entity due to an alteration in alveolar-pulmonary development. Material and Methods: A descriptive, retrospective study of newborns presenting CCAM in a tertiary care hospital in Madrid, Spain. Results: Seven patients were found. All were full term, normal weight births. Two patients showed respiratory distress at birth. Two chest x-rays were normal. CT scans showed three clear CCAM lesions, four suggestive of hybrid lesions. All were referred to medical centers with pediatric surgery for followup. Discussion: At birth, this pathology may be asymptomatic and appear as a chest $\mathrm{x}$-ray finding. Long term management is complicated by infection and malignization. Conservative treatment of asymptomatic patients includes regular follow up of lesions. Surgical treatment is reserved for symptomatic or complicated patients. Conclusions: Prenatal suspicion of CCAM is important since clinical exam and radiology may be normal in neonatal period. Chest CT scans are important in confirming diagnosis and determining future surgery. More studies are necessary for the proper diagnosis and management of this disorder.
\end{abstract}

(Key words: Alveolar development, congenital lung malformations, cystic adenomatoid malformation, CCAM, hybrid pulmonary lesion, newborn).

Rev Chil Pediatr 2011; 82 (2): 129-136

\section{RESUMEN}

Introducción: La malformación adenomatoidea quística (MAQ) es una entidad congénita poco frecuente debida a una alteración en el desarrollo alveolar pulmonar. Pacientes y Método: Se realizó un estudio descriptivo y retrospectivo de los recién nacidos con el diagnóstico prenatal de MAQ durante 6 años en un hospital terciario de Madrid (España). Resultados: Se encontraron un total de siete pacientes. Todos fueron recién nacidos a término de peso adecuado. Dos pacientes presentaron distress respiratorio al nacimiento. Dos radio-

Trabajo recibido el 17 de enero de 2011, devuelto para corregir el 22 de febrero de 2011, segunda versión el 28 de febrero de 2011, aceptado para publicación el 11 de abril de 2011.

Correspondencia a:

Pilar Antón-Martín.

E-mail: pilarantonmartin@hotmail.com 
grafías de tórax fueron normales. En la tomografía axial (TAC), tres lesiones fueron MAQ y cuatro fueron sugerentes de lesión híbrida. Todos se derivaron a centro con cirugía pediátrica para seguimiento. Discusión: Al nacimiento, esta patología puede permanecer asintomática y ser un hallazgo casual en una radiografía torácica. A largo plazo el riesgo de infección y malignización complican el manejo. El tratamiento conservador, que se dirige a pacientes asintomáticos, obliga a realizar controles seriados de las lesiones. El tratamiento quirúrgico se reserva para los pacientes con sintomatología o complicaciones postnatales. Conclusiones: El diagnóstico de sospecha prenatal de MAQ es fundamental dado que la clínica y radiología pueden ser normales en el período neonatal. Se debe realizar TAC torácico para confirmar la lesión y valorar futura cirugía. Se necesitan más estudios sobre el correcto diagnóstico y manejo de esta patología.

(Palabras clave: Malformaciones pulmonares congénitas; malformación adenomatoidea quística; MAQ; lesión pulmonar híbrida; recién nacido).

Rev Chil Pediatr 2011; 82 (2): 129-136

\section{Introducción}

Las malformaciones pulmonares congénitas constituyen una alteración en el desarrollo fetal de las diferentes estructuras pulmonares ${ }^{1}$. Dentro de estas anomalías congénitas, la malformación adenomatoidea quística (MAQ) afecta al desarrollo alveolar, determinando la formación de quistes que pueden ser diagnosticados ecográficamente en el período fetal. A pesar de su escasa incidencia, su diagnóstico debe tenerse en cuenta en el período neonatal ante la existencia de una dificultad respiratoria inmediata o una lesión radiográfica compatible en un paciente asintomático ${ }^{2}$. Por otro lado, aunque la mayoría de estas lesiones tienen buen pronóstico, su presencia requiere un seguimiento posterior del recién nacido afecto, para confirmar el diagnóstico prenatal y realizar tratamiento quirúrgico en el caso de que existan complicaciones asociadas. En otras ocasiones (pacientes asintomáticos) se prefiere un manejo postnatal más conservador, aunque este aspecto resulta más controvertido, ante el posible riesgo de futuras infecciones de repetición en la zona afecta o de malignización de la lesión ${ }^{3}$.

En el presente artículo presentaremos la experiencia de nuestro hospital en el diagnóstico, manejo y seguimiento posterior de esta patología, con especial énfasis en los hallazgos clínicos y radiológicos existentes en el período neonatal y realizaremos una revisión de la bibliografía existente, comparando nuestros resultados con los existentes en la literatura médica.

\section{Pacientes y Método}

Estudio descriptivo, retrospectivo, desde el 1 de enero de 2004 hasta el 31 de diciembre de 2009. Se analizaron las historias clínicas de los recién nacidos con el diagnóstico prenatal de esta malformación. Se valoró el momento del diagnóstico prenatal y el tipo de prueba de imagen realizada, las características del recién nacido (edad gestacional y peso al nacimiento), el tipo de parto y la sintomatología clínica y evolución postnatal inmediata de cada paciente. Se estudió también el tipo de malformación, los resultados de las pruebas complementarias, la presencia de otras malformaciones asociadas, el tratamiento recibido y su seguimiento posterior.

\section{Resultados}

En los seis años estudiados nacieron en nuestro centro 19180 niños, encontrándose 7 pacientes con diagnóstico prenatal de esta enfermedad (tabla 1), con una incidencia estimada de 1 malformación por cada 2740 recién nacidos vivos/año. Todos los diagnósticos antenatales se realizaron en el segundo trimestre de embarazo mediante ecografía obstétrica. El diagnóstico antenatal se completó con resonancia magnética fetal en 5 pacientes (figura 1).

Los 7 pacientes fueron recién nacidos a término con peso adecuado, con una mediana de edad gestacional de 39 semanas (rango 38-41) y de peso al nacimiento de 3006 gramos (rango 2 520-3 600). Cinco fueron varones frente 
Tabla 1. Hallazgos clínicos/radiográficos en el período neonatal de cada paciente

\begin{tabular}{|cccccclcc|}
\hline Caso & EG & Peso & Sexo & $\begin{array}{c}\text { Localización } \\
\text { lesión }\end{array}$ & $\begin{array}{c}\text { DRI al } \\
\text { nacimiento }\end{array}$ & Rx tórax & TAC tórax & $\begin{array}{c}\text { Malformación } \\
\text { asociada }\end{array}$ \\
\hline 1 & $38+1$ & 3260 & F & LID & No & Normal & Hibrido & No \\
\hline 2 & $40+1$ & 3035 & M & LII & No & Normal & Hibrido & No \\
\hline 3 & $39+1$ & 2520 & M & LID & No & Lesiones quísticas & MAQ & No \\
\hline 4 & $39+1$ & 3600 & M & LII & No & Lesiones quísticas & MAQ & No \\
\hline 5 & $40+5$ & 2690 & F & LII & No & Aumento densidad & MAQ & No \\
\hline 6 & $39+6$ & 2970 & M & L Medio & Si & Aumento densidad & Híbrido & Telangiectasia facial \\
\hline 7 & $38+6$ & 2970 & M & LID & Si & Lesiones quísticas & Hibrido & CIA mediana \\
\hline
\end{tabular}

* $\mathrm{MAQ}=$ malformación adenomatoidea quística, $\mathrm{EG}=$ edad gestacional, $\mathrm{DRI}=$ distrés respiratorio inmediato, $\mathrm{Rx}=$ radiografía, LID=lóbulo inferior derecho, LII=lóbulo inferior izquierdo, L Medio=lóbulo medio, M=masculino, F=femenino, CIA=comunicación interauricular.

a 2 mujeres. El tipo de parto fue eutócico en 3 pacientes, distócico vaginal (fórceps) en un paciente y cesárea en 3 pacientes. Las cesáreas se debieron a distocia por rotación, fracaso de inducción y electiva respectivamente. No se

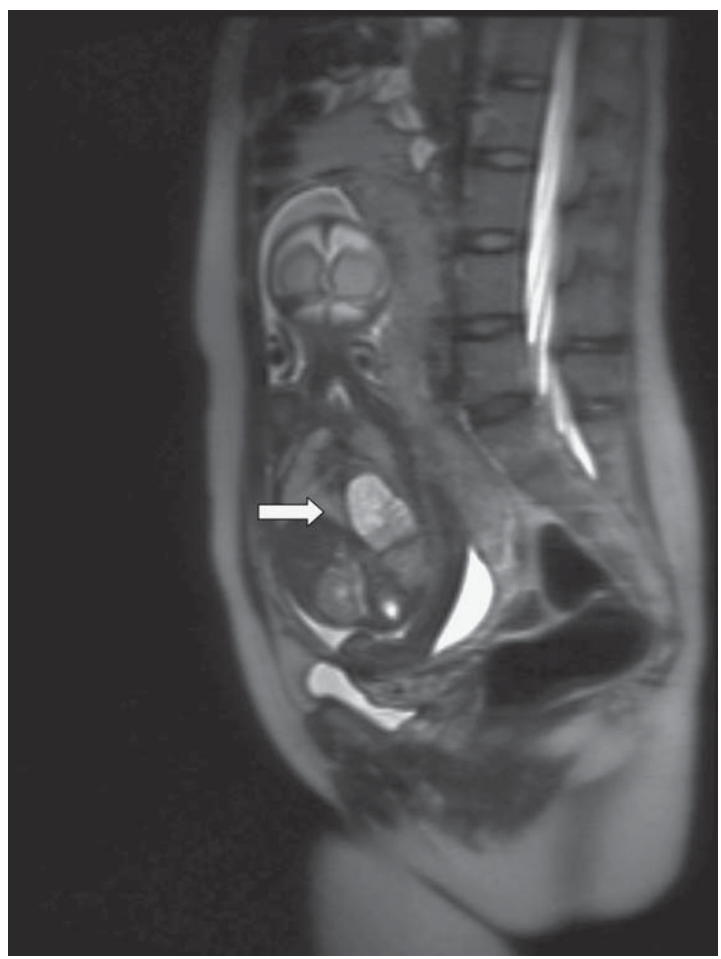

Figura 1. RMN fetal. Masa en hemitórax inferior izquierdo, bien delimitada, hiperintensa en secuencias T2, multiloculada, con cierto efecto de masa en probable relación con malformación quística. No identificamos vaso aberrante. realizó indicación específica de cesárea debido a la patología pulmonar existente.

$\mathrm{Al}$ nacimiento, 4 pacientes requirieron reanimación en sala de partos con oxígeno indirecto y 2 pacientes presentaron distress respiratorio inmediato leve que mejoró posteriormente con oxígenoterapia indirecta en incubadora. Uno de los pacientes con distress respiratorio presentó neumomediastino que se resolvió espontáneamente a las 24 horas del ingreso. Ninguno de los pacientes requirió ventilación mecánica ni presión positiva no invasiva.

En referencia a patologías asociadas a esta entidad se objetivó la existencia de una telangiectasia facial en uno de los pacientes, y una comunicación interauricular de $6 \mathrm{~mm}$ en otro, no encontrándose otras malformaciones congénitas ni patologías de interés en el resto de los casos analizados.

En cuanto a los hallazgos de imagen, la radiografía torácica realizada tras el nacimiento fue normal en 2 pacientes, describiéndose imágenes quísticas en 3 pacientes y aumento de densidad radiológica en los 2 pacientes restantes (figura 2). Seis de las 7 malformaciones se encontraron en lóbulos inferiores y una malformación se describió en lóbulo medio. Tres malformaciones fueron derechas, frente a 4 izquierdas. Para completar el estudio se realizó, en el primer mes de vida, Tomografía axial computarizada (TAC) torácica sin contraste endovenoso en todos los pacientes, que confirmó el diagnóstico en el 100\% de los casos, clasificándose las lesiones como MAQ en tres pa- 


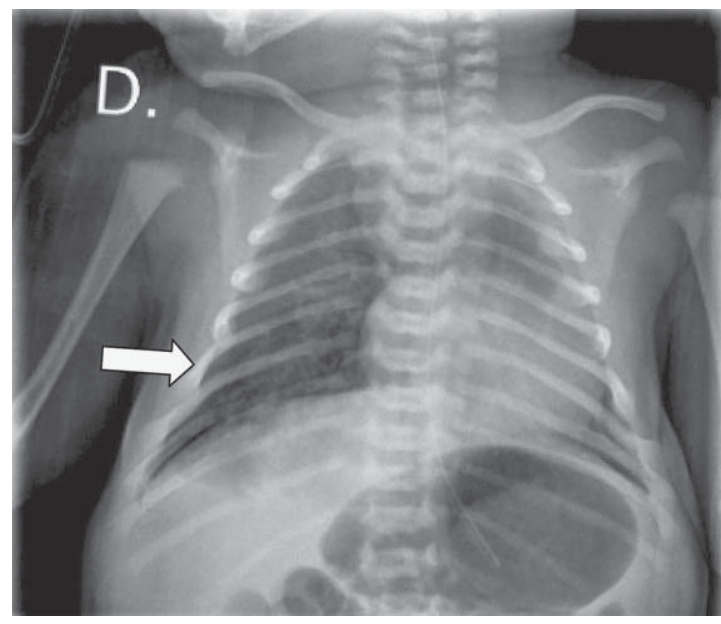

Figura 2. Rx tórax: Hemitórax derecho hiperclaro con neumomediastino y pequeñas burbujas aéreas, que desvía contralateralmente la silueta cardíaca, en relación con malformación congénita vía aérea pulmonar.

cientes y 4 sugerentes de lesión híbrida (MAQ/ secuestro pulmonar) (figura 3). No se realizó Angio Resonancia magnética nuclear (RMN) para determinar vascularización en ningún paciente. En la tabla 1 se resumen los hallazgos clínicos de nuestros pacientes y el correlato radiográfico de los mismos.

Una vez realizado el diagnóstico, y de forma programada, todos los pacientes fueron derivados a otro centro de referencia con cirugía pediátrica para su posterior seguimiento y valoración de tratamiento quirúrgico, dada la no existencia de cirugía en nuestro hospital.

\section{Discusión}

Las malformaciones pulmonares congénitas constituyen una alteración en el desarrollo embriológico y fetal de las distintas estructuras pulmonares. Suponen aproximadamente un 10\% de todas las malformaciones congénitas, siendo las más frecuentes aquellas que alteran el árbol bronquial, el parénquima pulmonar y las estructuras vasculares. Dependiendo de las estructuras afectadas, estas malformaciones congénitas se pueden clasificar en traqueobronquiales, bronquiales, pulmonares y

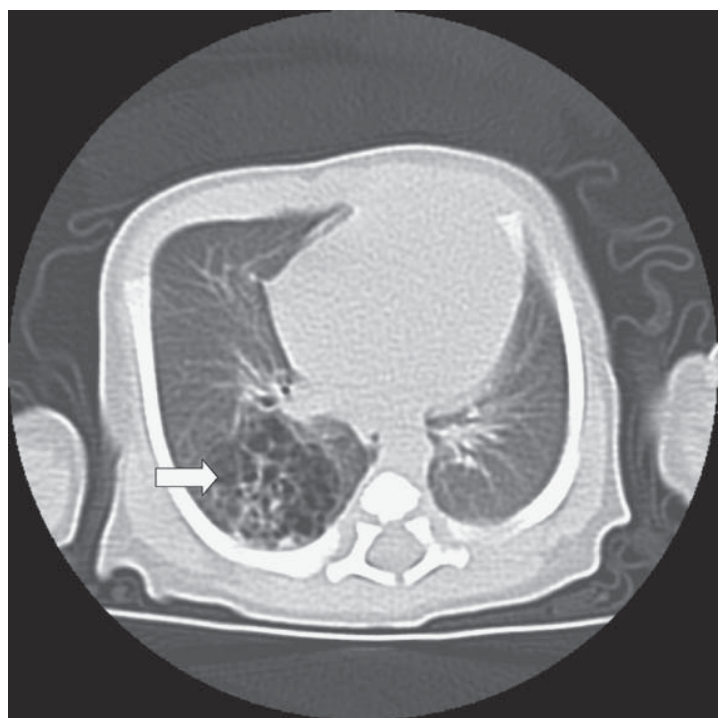

Figura 3. TAC torácico: Imágenes quísticas aéreas en LID $<1 \mathrm{~cm}$, con cierta expansión de dicho lóbulo, compatible con malformación pulmonar de vía aérea.

vasculares ${ }^{4}$. El diagnóstico prenatal ecográfico es difícil, pero importante al permitir elaborar una estrategia terapéutica y pronóstica para el futuro del recién nacido.

Dentro de estas malformaciones pulmonares congénitas las lesiones congénitas quísticas del pulmón (malformación adenomatoidea quística, secuestro pulmonar, enfisema lobar congénito y quiste broncogénico) presentan un origen embriológico y características clínicas similares. De todas estas lesiones, la más frecuente es la malformación adenomatoidea quística (MAQ) que se produce por un trastorno del desarrollo embrionario que impide la adecuada formación de alvéolos terminales, desarrollándose una zona pulmonar con aspecto quístico adenomatoso. Presenta comunicación con el árbol bronquial y recibe vascularización de la circulación pulmonar (aunque puede ser sistémica). Se asocia con relativa frecuencia a malformaciones cardiovasculares. Su incidencia aproximada es de 1 de cada 25 000-35 000 embarazos, siendo más frecuente en varones ${ }^{5}$. El secuestro pulmonar supone otro tipo de malformación pulmonar quística que se diferencia de la MAQ en su falta de comunicación con el árbol bronquial y su vascularización de origen sistémico y no pulmonar. En ocasiones 
MALFORMACIÓN QUÍSTICAS CONGÉNITAS

Tabla 2. Clasificación de las malformaciones adenomatoideas quísticas (Modificada de Stocker)

\begin{tabular}{|c|c|c|c|c|c|}
\hline Tipo & Tipo 0 & Tipo 1 & Tipo 2 & Tipo 3 & Tipo 4 \\
\hline Frecuencia & $1-3 \%$ & $>65 \%$ & $20-25 \%$ & $8 \%$ & $2-4 \%$ \\
\hline $\begin{array}{l}\text { Diámetro máximo } \\
\text { del quiste }\end{array}$ & $0,5 \mathrm{~cm}$ & $10 \mathrm{~cm}$ & $2,5 \mathrm{~cm}$ & $1,5 \mathrm{~cm}$ & $7 \mathrm{~cm}$ \\
\hline Células epiteliales & $\begin{array}{l}\text { Ciliado, pseudoes- } \\
\text { tratificado, columnar } \\
\text { alto, con células }\end{array}$ & $\begin{array}{l}\text { Ciliado, pseudoes- } \\
\text { tratificado, columnar } \\
\text { alto }\end{array}$ & $\begin{array}{l}\text { Ciliado, cuboidal } \\
\text { columnar }\end{array}$ & Ciliado, columnar & \\
\hline Células mucosas & $100 \%$ & $33 \%$ & No & No & No \\
\hline Músculo Liso & $100-500 \mathrm{mc}$ & $100-300 \mathrm{mc}$ & $50-100 \mathrm{mc}$ & $0-50 \mathrm{mc}$ & $25-100 \mathrm{mc}$ \\
\hline Cartílago & $100 \%$ & $5-10 \%$ & No & No & No \\
\hline Músculo estriado & No & No & $\mathrm{Si}$ & No & No \\
\hline
\end{tabular}

se observan lesiones pulmonares que presentan características radiológicas y histológicas comunes entre ambos tipos de malformaciones, dando lugar a las denominadas lesiones "híbridas", frecuentemente descritas ${ }^{9}$.

Desde la primera tipificación histológica de las MAQ, realizada en 1977 por Stocker et $\mathrm{al}^{10}$, se han desarrollado posteriormente otras clasificaciones basadas en el tamaño del quiste y en las características celulares (tabla 2). Actualmente, esta clasificación es la más utilizada aunque su aceptación no es universal, fundamentalmente por tres razones: existe cierta superposición entre los tipos, existen formas inclasificables y no es aplicable a los casos intervenidos durante el período fetal.

Desde el punto de vista clínico, esta patología puede manifestarse en el período neonatal con dificultad respiratoria inmediata o permanecer asintomática y ser un hallazgo casual en una radiografía torácica. A pesar de presentar escasa prevalencia en la edad infantil y adulta, se describen casos de pacientes con neumonías de repetición, neumotórax espontáneo, bronquitis obstructiva o dolor torácico debidas a la existencia de este tipo de malformación. Cuando se diagnóstica durante la gestación, su principal problema reside en el tamaño de la lesión. Si ésta es muy grande puede producir desplazamiento mediastínico, compresión esofágica, polihidramnios y finalmente hidrops fetal, poniendo en peligro la vida del futuro recién nacido. Tras el nacimiento, la inestabilidad respiratoria por el gran tamaño de la lesión, el riesgo de neumonías de repetición y la posibilidad de futura degeneración maligna (tasa anual del 3\%) complican el pronóstico de estos pacientes ${ }^{11}$.

El diagnóstico prenatal se realiza mediante ecografía en el segundo trimestre de embara$\mathrm{zo}^{12-13}$, y es aconsejable confirmarlo con resonancia magnética fetal que identifica con más precisión las estructuras anatómicas afectadas y evalúa la coexistencia de otras anomalías congénitas asociadas ${ }^{14,15}$. Típicamente estas lesiones presentan un incremento del tamaño con un pico máximo a las 28 semanas, con estabilización e incluso regresión posterior, estimándose un 15\% de casos de desaparición de la malformación intraútero durante el tercer trimestre ${ }^{16}$.

En el período postnatal la radiografía de tórax puede mostrar varios tipos de lesiones, siendo la más frecuente la presencia de imágenes quísticas ${ }^{17}$. El TAC torácico confirma el diagnóstico, la extensión de la lesión, su asociación con otras malformaciones no pulmonares y permite diferenciarla de otras anomalías pulmonares como el secuestro pulmonar, el quiste broncogénico, el enfisema lobar congénito los neumatoceles y las bronquiectasias ${ }^{18}$. La angio-resonancia magnética determina la dependencia vascular de la lesión. Ambas técnicas son de gran utilidad de cara a la futura cirugía.

A pesar de los nuevos avances, la tipificación de las lesiones por técnicas radiográficas tiene poca relación con los hallazgos histo- 
lógicos de las lesiones posnatales extirpadas quirúrgicamente. En algunas publicaciones se ha identificado coexistencia de varios tipos de malformaciones pulmonares o incluso varios subtipos de MAQ en el mismo paciente, sugiriéndose un origen embriológico común de todas ellas ${ }^{19}$.

Existen diferentes abordajes terapéuticos dependiendo del tamaño de la lesión y de la edad al diagnóstico de la misma. En el período prenatal se han realizado drenaje, ablación percutánea o resección intraútero en aquellos fetos con quistes grandes y riesgo de desarrollo de hidrops fetalis, con resultados aceptables según las series ${ }^{20-23}$. Existe controversia respecto al uso de corticoides antenatales, aunque existen revisiones de casos donde se encuentra regresión de aquellas lesiones con tamaño microquístico $^{24,25}$. En el manejo postnatal existen dos tendencias. Por una parte, el tratamiento conservador, que se dirige a los pacientes asintomáticos. En ellos, controles seriados de las lesiones son obligados, para determinar su posible degeneración maligna. El tratamiento quirúrgico se reserva para los pacientes con sintomatología o complicaciones postnatales ${ }^{26,28}$, aunque existen autores que defienden la cirugía universal ${ }^{29,30}$, e incluso precoz ${ }^{31,32}$, a pesar de que el paciente permanezca asintomático, dadas las posibles complicaciones posteriores. El tipo de resección depende del tamaño de las lesiones, habiéndose encontrado resultados similares en cuanto a supervivencia y complicaciones con lobectomía vs segmentectomía en lesiones de pequeño tamaño ${ }^{33}$. El tipo de abordaje suele ser la toracotomía, aunque se describen buenos resultados en lesiones de pequeño tamaño con videotoracoscopia ${ }^{34,35}$.

En el análisis detallado de nuestros resultados cabe destacar la elevada incidencia encontrada en nuestra serie (una malformación por cada 2740 recién nacidos y año) comparada con la existente en la literatura. Este dato llama más la atención si tenemos en cuenta que nuestro centro, un hospital terciario situado en Madrid (España), está capacitado para la atención intensiva del recién nacido, pero carece de cirugía pediátrica, y no es por tanto un centro de referencia en patología congénita de diagnóstico prenatal. Es posible que nuestros datos se aproximen más a la incidencia real que podría estar subestimada. La inexistencia de sintomatología en numerosos recién nacidos, el hecho de que muchas de estas malformaciones se diagnostiquen en edades más tardías, y la mejora en el seguimiento prenatal con ecografía e incluso RMN, está permitiendo el diagnóstico de numerosas entidades que previamente pasaban desapercibidas. Sin embargo, no podemos descartar que existan otros factores de riesgo, por el momento desconocidos, que contribuyan al aumento de las tasas de incidencia de esta enfermedad en nuestra población. En referencia al sexo, la mayor prevalencia de varones descrita en diferentes series también se recoge en nuestro centro. Por otro lado, a pesar de que en todos los trabajos se recoge la asociación de estas malformaciones con cardiopatías congénitas en ocasiones complejas, en nuestra serie sólo hemos encontrado un paciente que presentaba una comunicación interauricular hemodinámicamente poco significativa asociada a esta entidad. Al igual que se recoge en numerosas publicaciones, la ausencia de clínica en el recién nacido y de hallazgos radiográficos al ingreso es un dato frecuente y que hemos constatado en nuestra revisión. Así, 5 pacientes no presentaron síntomas respiratorios al nacimiento y 2 tuvieron controles radiológicos normales. Estos resultados nos deben hacer reflexionar sobre la necesidad de realizar un estudio más exhaustivo por TAC torácico en edades tempranas para el correcto diagnóstico de esta patología y establecer el mejor seguimiento posterior.

\section{Conclusiones}

En el manejo de las malformaciones pulmonares congénitas en el período neonatal es fundamental el diagnóstico de sospecha prenatal ya que al nacimiento el paciente puede estar asintomático desde el punto de vista respiratorio y/o con radiografía de tórax normal. Es de uso obligado el realizar un TAC torácico para confirmar el diagnóstico y determinar la situación anatómica de las lesiones de cara a la posible necesidad de intervención quirúrgica. Se necesitan más estudios para determinar si 
se debe realizar cirugía en los pacientes asintomáticos, en qué momento y bajo qué tipo de técnica.

\section{Referencias}

1.- Escobar LF, Fuentes DF, Urzúa FC, Capetillo FM: Malformación adenomatoidea quística pulmonar de presentación tardía: revisión del tema y presentación de un caso. Rev Chil Radiol 2010; 16: 190-4.

2.- Langston C: New concepts in the pathology of congenital lung malformations. Semin Pediatr Surg 2003; 12: 17-37.

3.- Astorga L, Herrera $O$, Webar E, et al: Nota radiológica. Caso radiológico pediátrico. Rev Chil Enf Respir 2003; 19: $166-8$.

4.- Carrera M, Núñez B: Enfermedades pulmonares congénitas. Medicine 2006; 63: 4105-9.

5.- Perdomo J, Domínguez M, Núñez A, Luna E: Prenatal diagnosis of lung cystic adenomatoid malformation: About 2 cases. Rev Cubana Genet Comunit 2009; 3: 54-7.

6.- Griffin N, Devaraj A, Goldstraw P, Bush A, Nicholson AG, Padley S: CT and histopathological correlation of congenital cystic pulmonary lesions: a common pathogenesis. Clin Radiol 2008; 63: 995-1005.

7.- Wilson RD, Hedrick HL, Liechty KW, et al: Cystic adenomatoid malformation of the lung: review of genetics, prenatal diagnosis, and in utero treatment. Am J Med Genet 2006; 140: 151-5.

8.- Correia-Pinto J, Gonzaga S, Huang Y, Rottier R: Congenital lung lesions--underlying molecular mechanisms. Semin Pediatr Surg 2010; 19: 171-9.

9.- Chen HW, Hsu WM, Lu FL, et al: Management of congenital cystic adenomatoid malformation and bronchopulmonary sequestration in newborns. Pediatr Neonatol 2010; 51: 172-7.

10.- Stocker JT, Madewell JE, Drake RM: Congenital cystic adenomatoid malformation of the lung. Classification and morphologic spectrum. Hum Pathol 1977; 8: 15571.

11.- Laberge JM, Puligandla P, Flagole H: Asymptomatic congenital lung malformations. Semin Pediatr Surg 2005; 14: 16-33.

12.- Chow PC, Lee SL, Tang MH, et al: Management and outcome of antenatally diagnosed congenital cystic adenomatoid malformation of the lung. Hong Kong Med J 2007; 13: 31-9.

13.- Sauvat F, Michel JL, Benachi A, Emond S, Revillon Y:
Management of asymptomatic neonatal cystic adenomatoid malformations. J Pediatr Surg 2003; 38: 548-52.

14.- Hubbard AM, Adzick NS, Crombleholme TM, et al: Congenital chest lesions: diagnosis and characterization with prenatal MR imaging. Radiology 1999; 212: 43-8.

15.- Liu YP, Chen CP, Shih SL, Chen YF, Yang FS, Chen SC: Fetal cystic lung lesions: evaluation with magnetic resonance imaging. Pediatr Pulmonol 2010; 45: 592-600.

16.- Mann S, Wilson RD, Bebbington MW, Adzick NS, Johnson MP: Antenatal diagnosis and management of congenital cystic adenomatoid malformation. Semin Fetal Neonatal Med 2007; 12: 477-81.

17.- Jana M, Gupta AK: Radiologic evolution of congenital cystic adenomatoid malformation in a neonate. Indian J Pediatr 2010; 77: 212-3.

18.- Calvert JK, Boyd PA, Chamberlain PC, Syed S, Lakhoo $K$ : Outcome of antenatally suspected congenital cystic adenomatoid malformation of the lung: 10 years' experience 1991-2001. Arch Dis Child Fetal Neonatal Ed 2006; 91: 26-8.

19.- Tomita SS, Wojtczak H, Pickard R, Vázquez WD: Congenital cystic adenomatoid malformation and bronchogenic cyst in a 4-month-old infant. Ann Thorac Cardiovasc Surg 2009; 15: 394-6.

20.- Nicolai T: Management of the upper airway and congenital cystic lung diseases in neonates. Semin Fetal Neonatal Med 2009; 14: 56-60.

21.- Liechty KW: Ex-utero intrapartum therapy. Semin Fetal Neonatal Med 2010; 15: 34-9.

22.- Mann S, Johnson MP, Wilson RD: Fetal thoracic and bladder shunts. Semin Fetal Neonatal Med 2010; 15: 28-33.

23.- Sepúlveda W, Mena F, Ortega X: Successful percutaneous embolization of feeding vessels of a lung tumor in a hydropic fetus. J Ultrasound Med 2010; 29: 639-43.

24.- Morris LM, Lim FY, Livingston JC, Polzin WJ, Crombleholme TM: High-risk fetal congenital pulmonary airway malformations have a variable response to steroids.J Pediatr Surg 2009; 44: 60-5.

25.- Curran PF, Jelin EB, Rand L, et al: Prenatal steroids for microcystic congenital cystic adenomatoid malformations. J Pediatr Surg 2010; 45: 145-50.

26.- Hammond PJ, Devdas JM, Ray B, Ward-Platt M, Barrett $A M$, McKean M: The outcome of expectant management of congenital cystic adenomatoid malformations (CCAM) of the lung. Eur J Pediatr Surg 2010; 20: 145-9.

27.- Liao SL, Lai SH, Hsueh C, Wong KS: Comparing late-onset and neonatally-diagnosed congenital cystic adenomatoid malformation of the lung. Chang Gung Med J 2010; 33: 36-43. 
28.- Burge $D$, Wheeler $R$ : Increasing incidence of detection of congenital lung lesions. Pediatr Pulmonol 2010; 45: 103.

29.- Lakhoo K: Management of congenital cystic adenomatous malformations of the lung. Arch Dis Child Fetal Neonatal Ed 2009; 94: 73-6.

30.- Wong A, Vieten D, Singh S, Harvey JG, Holland AJ: Long-term outcome of asymptomatic patients with congenital cystic adenomatoid malformation. Pediatr Surg Int 2009; 25: 479-85.

31.- Kim YT, Kim JS, Park JD, Kang CH, Sung SW, Kim $J H$ : Treatment of congenital cystic adenomatoid malformation-does resection in the early postnatal period increase surgical risk? Eur J Cardiothorac Surg 2005; 27: 658-61.
32.- Komori K, Kamagata S, Hirobe S, et al: Radionuclide imaging study of long-term pulmonary function after lobectomy in children with congenital cystic lung disease. J Pediatr Surg 2009; 44: 2096-100.

33.- Kim HK, Choi YS, Kim K, et al: Treatment of congenital cystic adenomatoid malformation: should lobectomy always be performed? Ann Thorac Surg 2008; 86: 24953.

34.- Kaneko K, Ono Y, Tainaka T, Sumida W, Kawai Y, Ando $H$ : Thoracoscopic lobectomy for congenital cystic lung diseases in neonates and small infants. Pediatr Surg Int 2010; 26: 361-5.

35.- Bush A: Prenatal presentation and postnatal management of congenital thoracic malformations. Early Hum Dev 2009; 85: 679-84. 